Rev. Elev. Méd. vét. Pays trop., 1972, 25 (2) : 155-159

\title{
Transmission de la peste bovine par des veaux possédant une immunité maternelle résiduelle
}

\author{
par A. PROVOST (*) \\ (avec la collaboration technique de Mme G. DUFAU)
}

\begin{abstract}
RESUME
Des veaux possédant un reliquat d'immunité colostrale, infectés expérimentalement par voie nasale avec un virus bovipestique, ont contaminé des veaux sensibles placés à leur contact sans qu'il paraisse y avoir eu de multiplication du virus chez les premiers. Un veau a fait une forme pulmonaire de longue durée, assimilable à un portage chronique.
\end{abstract}

La possibilité qu'ont des bovins vaccinés contre la peste bovine de transmettre le contage bovipestique après qu'ils aient été euxmêmes contaminés par cohabitation avec des malades est une notion nouvelle pour la compréhension de l'épizootiologie de la peste bovine (6). Elle a eu pour conséquence la découverte de l'absence d'activité neutralisante du virus pestique dans le mucus nasal des bovins vaccinés et des veaux encore sous couvert de l'immunité colostrale (4), et, en application pratique, la proposition de vaccination des veaux par voie pernasale (5).

A la réflexion, on devait se demander quel rôle pouvaient jouer les veaux, passivement immuns après absorption du colostrum maternel, dans l'épizootiologie de la peste; en effet, leur statut immunitaire est, pendant les quelques mois où ils hébergent des anticorps sériques d'origine colostrale, semblable à celui d'adultes immunisés activement: présence d'anticorps neutralisants dans leur plasma mais absence dans le mucus nasal, ce qui laisse une voie d'entrée à un virus de contamination. La présente expérience a été montée pour tirer cette affaire au clair.

(*) I.E.M.V.T., Laboratoire de Farcha, B.P. no 433, Fort-Lamy, Tchad.

\section{MATERIEL ET METHODES}

Le protocole expérimental est simple: disposer de veaux à immunité antipestique colostrale, les contaminer avec un virus bovipestique puis, en un temps ultérieur, placer à leur contact des bovins sensibles à la peste et observer une éventuelle transmission du contage.

\section{Animaux d'expérience}

On achète chez un éleveur 7 veaux âgés de 3 à 4 mois et l'on vérifie qu'ils possèdent des anticorps sériques antipestiques qui, de par leur âge et leur origine, ne peuvent être que d'origine colostrale.

Deux bouvillons d'un an environ, sans cicatrice auriculaire de vaccination, entrent ainsi en expérience; on s'assure que leur sérum n'héberge aucune activité neutralisante antipestique. Ils sont placés au contact des 7 premiers veaux 24 heures après l'infection de ces derniers et après que ceux-ci aient été lavés avec une solution antiseptique (D 39) pour enlever toute trace de virus sur leur pelage.

Des prises de sang et des collectes de mucus 
nasal sur écouvillon de coton sont réalisées à la demande.

Les animaux sont maintenus en cohabitation pendant toute la durée de l'expérience.

\section{Virus bovipestique}

Rate de bouvillon naturellement infecté dans le village de Madjorio, abattu en phase hyperthermique au stade des lésions muqueuses débutantes; il n'a été procédé à aucun titrage $\mathrm{du}$ virus.

On effectue un broyat de rate au 1/10 en solution physiologique et on dépose $1 \mathrm{ml} \mathrm{du}$ surnageant de centrifugation dans les cavités nasales de chacun des 7 veaux à l'aide d'une sonde souple à bords mousses.

\section{Techniques sérologiques}

Ce sont celles qui sont classiquement appliquées : a) séro-neutralisation quantitative suivant la méthodologie de PLOWRIGHT et FERRIS (3).

b) muco-neutralisation qualitative, selon un protocole déjà décrit (4); étant donné les conditions d'expérience, le résultat fournit une réponse positive ou négative quant à une activité neutralisante du mucus, sans qu'on puisse la quantifier.

c) immunofluorescence qualitative pour la recherche des anticorps pestiques dans le mucus par une méthode indirecte (technique du "sandwich ") déjà décrite (4).

d) précipitation. Diffusion en gélose mettant en œuvre la technique décrite par SCOTT et BROWN (9).

\section{RESULTATS}

Le tableau I collige la marche et les résultats de l'expérience.

TABLEAU $\mathrm{N}^{\circ} \mathrm{I}$

Cinëtique sérologique de veaux à immunitê antipestique colostrale infectês de peste par voie nasale et de veaux réceptifs placés à leux contact.

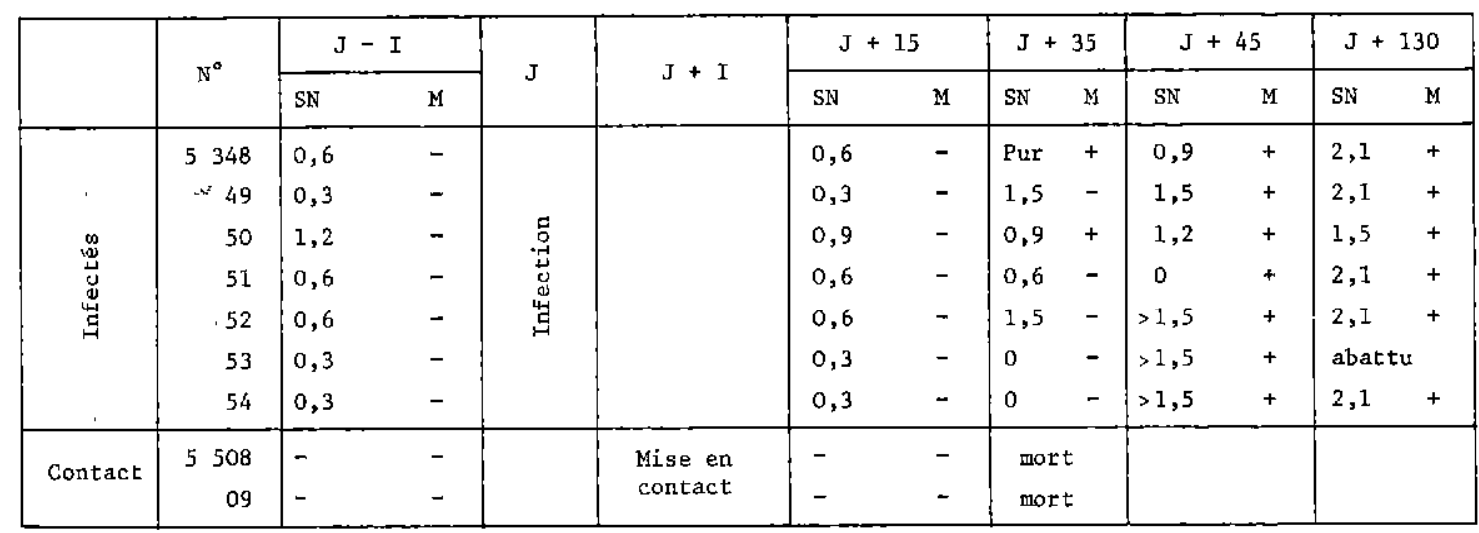

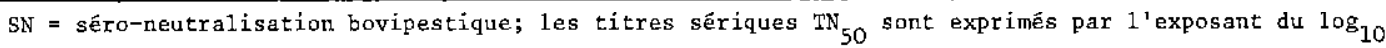
de la dilution de sérum. $M=$ muco-neutralisation qualitative.

\section{Evolution clinique}

Les veaux infectés de peste bovine par voie nasale n'ont extériorisé aucun symptôme morbide pendant les trois premières semaines qui ont suivi l'infection.

Par contre, les bouvillons 5508 et 5509 placés à leur contact montrent des signes cliniques les $18^{\mathrm{e}}$ et $20^{\mathrm{e}}$ jours et meurent après une maladie classique.

Le $22^{\mathrm{e}}$ jour après la mise en contact, le veau 5353 débute une peste qui n'a chez lui qu'une évolution avortée : fièvre à $40^{\circ} \mathrm{C}$ pendant une semaine, inappétence, larmoiement, petites ulcérations buccales. 
Tout est rentré dans l'ordre le $30^{\mathrm{e}}$ jour. On remarque néanmoins que l'animal conserve un poil piqué et tousse de temps à autre. Inquiété par ces accès de toux et suspectant une maladie pulmonaire, l'expérimentateur décide le sacrifice le $45^{\mathrm{e}}$ jour après la mise en contact. A l'autopsie, on note un foyer d'hépatisation du lobe apical droit, de couleur rouge carminée, sec à la coupe, à démarcation nette avec le parenchyme pulmonaire non atteint.

Une coupe histologique colorée par l'hémalun-éosine montre l'existence d'une bronchopneumonie de type viral avec infiltration cellulaire des septums alvéolaires et, surtout, la présence de nombreux plasmodes multinucléés présentant des inclusions cytoplasmiques et nucléaires éosinophiles (photos).

Un doute naissant, on réalise une précipitation en gélose avec un broyat de ganglion bronchique qui s'avère être positive avec un sérum bovipestique précipitant. Du broyat de la lésion pulmonaire, on isole un virus bovipestique en cultures cellulaires.

Les autres veaux n'ont, quant à eux, extériorisé aucun signe clinique.

\section{Cinétique sérologique}

Pendant les 15 jours qui ont suivi leur infection par voie nasale, les anticorps sériques neutralisants des veaux n'ont pas été modifiés hormis ceux du 5350 qui ont accusé une légère chute. Fait remarquable, les muco-anticorps ne sont pas non plus apparus.

Ce n'est qu'au contrôle du $35^{\circ}$ jour qu'ils apparaissent chez deux animaux (5348 et 5350), tandis que la sérologie sérique est variable, décroissante chez les uns $(5348,5353$ et $5354)$, stable $(5550,5551)$ ou en augmentation pour d'autres $(5349,5552)$. Il paraît intéressant de noter que le veau 5353 qui était, ce jour-là, à son $13^{e}$ jour de maladie ait encore une sérologie négative alors qu'il est classique qu'à cette période on détecte des anticorps neutralisants à un titre élevé (8). On est réduit aux conjectures: déficience du système immunoformateur, ce qui paraît improbable au regard des résultats ultérieurs, ou présence de virus, inhabituelle, dans le sérum sanguin, hypothèse elle aussi difficilement acceptable.
$\mathrm{Au} 45^{\mathrm{e}}$ jour, un seul veau (5351) est négatif. Il élaborera plus tard ses anticorps sériques car dès ce jour on détecte chez lui, comme chez tous les autres, des muco-anticorps nasaux.

A la fin de l'expérience, tous les veaux ont une solide immunité, générale et locale.

\section{DISCUSSION}

L'expérience vaut tout d'abord d'être jugée dans son ensemble.

Globalement, un ensemble de veaux possédant une immunité colostrale antipestique évanescente peut servir de relais à un virus pestique de contamination, vraisemblablement par la muqueuse nasale, et devenir à son tour foyer d'infection disséminateur du virus.

Lorsque l'immunité colostrale résiduelle est assez forte, il n'y a pas de séro-conversion pestique. Ce fait, ajouté à l'absence de mucoanticorps jusqu'aux alentours du $20^{\circ}$ jour après l'infection, au minimum, conduit à penser que le virus de contamination ne s'est pas répliqué chez ces veaux; ils n'ont joué qu'un rôle de vecteurs passifs.

Cependant, au fur et à mesure que décrô̂t l'immunité colostrale et si l'apport infectieux se poursuit (ce qui est le cas puisque les deux veaux 5508 et 5509 , ayant contracté la peste, ont été maintenus en contact avec leurs congénères), on assiste à la génèse d'une immunité active locale et générale sans que l'on note pour autant d'infection cliniquement décelable. Ainsi se crée un foyer occulte de peste bovine dont le résultat est l'immunisation des animaux.

Cette expérience toute simple est ainsi riche d'enseignements. Elle apporte une explication à l'apparition inopinée de foyers de peste dans des troupeaux de veaux d'âges variables comme on les rencontre dans le Sahel. Elle satisfait l'esprit en indiquant l'origine des anticorps neutralisants de haut titre que l'on rencontre chez les bouvillons non vaccinés (7): on pouvait se douter du processus mais il n'avait pas été démontré. Il n'y a pas lieu, pour l'expliquer, d'invoquer l'existence d'une souche hypo-virulente : la séro-conversion s'établit au contact de malades lorsque disparaissent les anticorps colostraux. Si ces derniers sont disparus depuis 
quelques semaines, il y a maladie clinique, ce qui a été le cas des veaux témoins mis en contact.

C'est à cette conclusion qu'arrivait CURASSON (2), il y a plusieurs décennies, à la suite d'observations faites sur le terrain: "c'est après le sevrage et alors que l'immunité acquise par le jeune va rapidement décroissant, que l'infection naturelle peut donner une forme atténuée vaccinante $\%$.

Le cas du veau 5353 est lui aussi digne d'intérêt. Il est difficile d'opter pour une peste à incubation prolongée sous couvert de l'immu- nité colostrale résiduelle comme cela a été rapporté par d'anciens observateurs (1) ou pour une réinfection dans le groupe; le point n'a d'ailleurs pas de portée dans la présente observation. Ce qui est remarquable, c'est que cet animal ait fait une peste dont il a guéri, qu'il ait élaboré des anticorps sériques neutralisants et que néanmoins il ait conservé un foyer infectieux profond.

On confirme ainsi expérimentalement l'opinion, soutenue par CURASSON (2), de l'existence de porteurs de virus dans la peste bovine; s'ils sont certes rares, il se peut néanmoins qu'ils existent en région d'enzootie.

\section{SUMMARY}

Rinderpest transmission by calves with maternal residual colostral immunity

Calves with residual colostral immunity have been experimentally infected with rinderpest virus via the nasal passage; they have contaminated in contact susceptible cattle without evidence of virus multiplication. One calf has presented a long-lasting pulmonary form recalling a chronic carriage.

\section{RESUMEN}

\section{Transmisión de la peste bovina por terneros teniendo una inmunidad maternal residual}

Terneros teniendo un residuo de inmunidad calostral, experimentalmente infectados por via nasal con un virus bovipestico, contaminaron terneros sensibles puestos en contacto con ellos, sin multiplicación evidente del virus. Un ternero presentó una forma pulmonar de larga duración, como un portador de gérmenes crónico.

\section{BIBLIOGRAPHIE}

1. AUFFRET et DAI, cités par Curasson (3).

2. CURASSON (G.), Traité de pathologie exotique vétérinaire et comparée, 2 e éd., tome 1, Paris, Vigot Frères, 1942.

3. PLOWRIGHT (W.) et FERRIS (R.D.), Studies with rinderpest virus in tissue-culture. III. The stability of culture virus and its use in virus neutralization tests, Arch. ges. Virusf., 1961, 11 : 516-33.

4. PROVOST (A.), Observations sur les mucoanticorps nasaux des bovins, Rev. Elev. Méd. vét. Pays trop., 1970, $23: 289-93$.

5. PROVOST (A.), BORREDON (C.), Essais de vaccination antipestique par voie pernasale de veaux possédant ou non une immunité colostrale, Rey. Elev. Méd. vét. Pays trop., 1972, 25 (2).
6. PROVOST (A.), MAURICE (Y.) et BORREDON (C.), Comportement clinique et immunologique lors de contamination bovipestique de bovins vaccinés depuis plusieurs années avec un vaccin antipestique de cultures cellulaires, Rev. Elev. Méd. vét. Pays trop., 1969, 22: 453-64.

7. Rapport annuel du Laboratoire de Farcha pour 1969 , tome I, p. 61.

8. SCOTT (G. R.), Diagnosis of rinderpest. Rome, Food and Agricultural Organization, 1967 (FAO Agricultural studies $n^{\circ} 71$ ).

9. SCOTT (G. R.), BROWN (R.D.), Rinderpest diagnosis with special reference to the agar gel double diffusion test, Bull. epizoot. Dis. Afr., 1961, 9 : 83-125. 


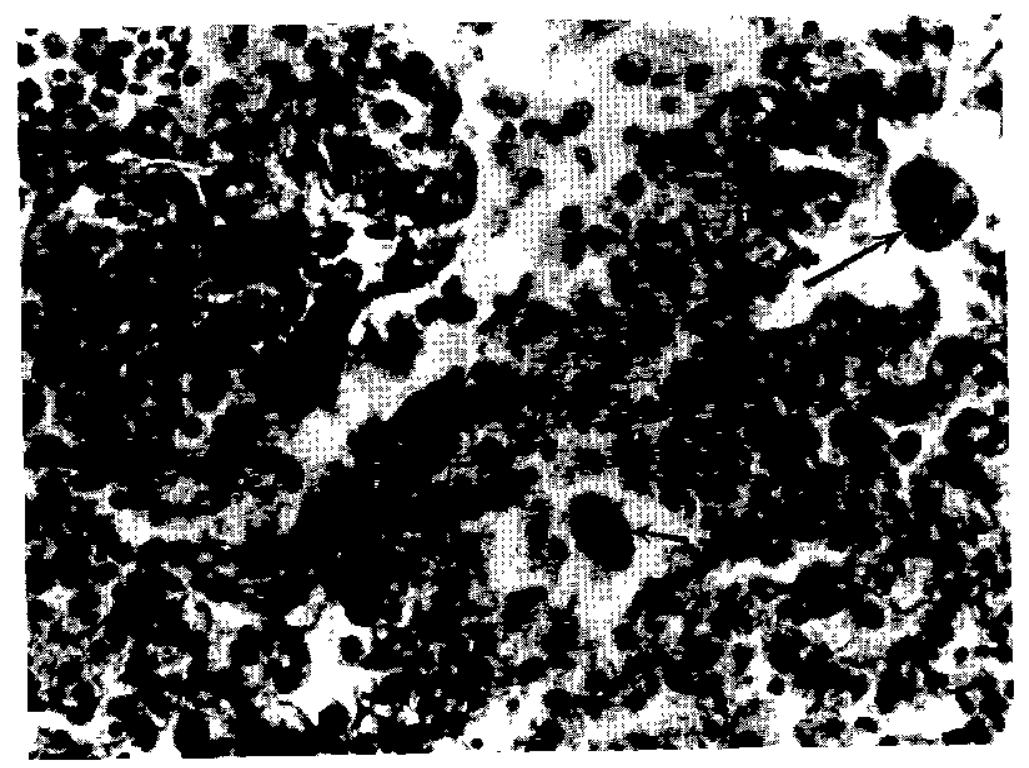

Photo I A. - Veau 5353. Image histopathologique d'une coupe de lésion pulmonaire. Noter la présence de plusieurs polycaryocytes. $G=200$.

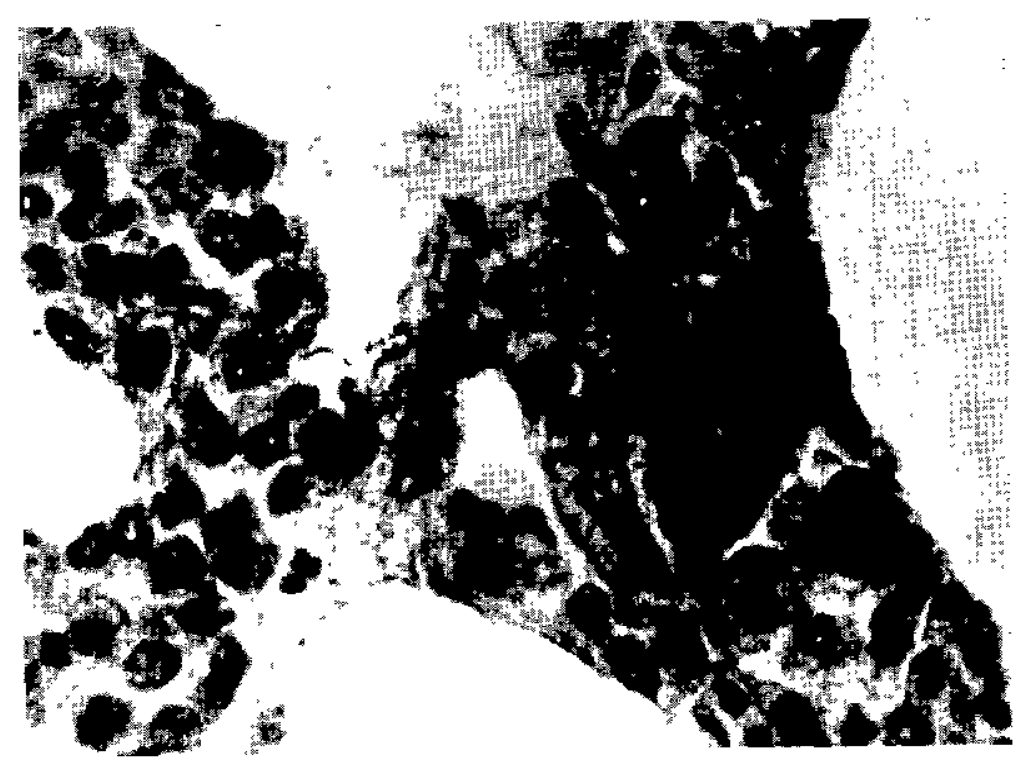

Photo I B. - Veau 5353. Même orıgıne. Noter l'infiltration des parois alvéolaires et la présence diun plasmode multinucléé (à droite). $\mathrm{G}=500$. 